\title{
Um Modelo para Interações entre Quarks: A Redução Não-Relativística em Modelos de Potenciais
}

\author{
A Model for Quark Interactions: The Nonrelativistic Reduction in Potential Models \\ Alex Eduardo de Bernardini \\ Departamento de Raios Cósmicos, IFGW, UNICAMP, \\ C.P. 6165, 13083-970, Campinas, São Paulo, Brasil \\ E-mail: alexeb@ifi.unicamp.br.
}

Recebido em 17 de abril, 2003. Aceito em 08 de agosto, 2003.

\begin{abstract}
Modelos fenomenológicos de potenciais têm proporcionado resultados extremamente satisfatórios na descrição dos hádrons ordinários, mais especificamente no caso dos estados ligados quark-antiquark (mésons). Neste trabalho estudamos os principais aspectos na construção das interações em um estado ligado quark-antiquark proporcionando a obtenção das ferramentas genéricas para o estudo de modelos de potenciais. Analisamos os aspectos importantes na escolha do tratamento de maior concordância com a dinâmica de interação das partículas, considerando os aspectos relativísticos bem como as possibilidades de uma aproximação não-relativística. Inicialmente a cromodinâmica quântica (QCD) "macia" é empregada para determinar os termos do potencial efetivo estabelecendo o termo assintótico Coulombiano decorrente da aproximação pela troca de um glúon. Ao mesmo tempo, um termo linear de confinamento é introduzido em concordância com a QCD e com a prescrição fenomenológica. Finalizando, discutimos as possibilidades gerais para o desenvolvimento de um modelo de potencial.

Phenomenological potential models have provided extremely satisfactory results on description of ordinary hadrons, more specifically in the case of quark-antiquark bound states (mesons). In this work we study the main aspects in the construction of quark-antiquark interactions just providing the generic tools for potential model study. We analyze the important aspects in the choice of the treatment in good agreement with the dynamics of interacting particles, attempting to relativistic aspects as well as to the possibilities of nonrelativistic approximation. Initially the "soft QCD" is employed to determine the effective potential terms establishing the asymptotic Coulomb term from one gluon exchange approximation. At the same time, a linear confinement term is introduced in accordance with QCD and phenomenological prescription. At the end, we discuss the general developing possibilities of a potential model.
\end{abstract}

\section{Introdução}

A idéia de que os hádrons são compostos por partículas ainda mais fundamentais tem uma longa história. Em 1949, Fermi e Yang [1] propuseram uma idéia interessante do píon (méson $\pi$ ) como um estado ligado do próton (p) e do nêutron (n) em um multipleto do $S U(2)$. No início dos anos 50, de maneira a explicar a regra de Nakano, Nishijima e GellMann (NNG) [2, 3], a conservação de isospin (I) e de estranheza (S), Sakata [4] estendeu a idéia de Fermi e Yang para hádrons incluindo partículas estranhas, considerando $\mathrm{p}, \mathrm{n}$ e a partícula $\Lambda$ como partículas fundamentais. Apesar do modelo corretamente formulado segundo uma álgebra de Lie com simetria unitária, algumas dificuldades já se apresentavam, como o fato de se prever spin $\frac{1}{2}$ para a partícula $\Xi$, diferente do valor experimental medido $\frac{3}{2}$, ou o fato do modelo não permitir a construção do octeto de bárions. Foi em 1964 que, então, Gell-Mann e Zweig [5, 6, 7], independentemente, propuseram o modelo a quarks [8]: o quark seria considerado como uma partícula sub-hadrônica e do ponto de vista teórico seria uma realização da representação fundamental da álgebra de Lie do $S U(3)$.

Depois disso tivemos a descoberta do charmônio em 1974 [9, 10] e, na mesma década, o advento da Cromodinâmica Quântica (QCD) [11-14] que é a teoria que descreve a dinâmica de quarks e glúons.

No Brasil, nos anos 70-80, o estudo de modelos efetivos para interações hadrônicas se caracterizava como um tópico de pesquisa bastante ativo. Dentre outros, destacamos os trabalhos de P. Leal Ferreira et al. [15-17] bem como a tese de doutoramento de F. M. Witkowski [18, 19] onde propriedades eletromagnéticas de hádrons são estudadas em um modelo relativístico.

Deste período em diante desenvolveram-se técnicas marcantes para se utilizar cálculos perturbativos [13, 14, 20] no tratamento da "dinâmica de cor". Contudo, é muito difícil se obter as propriedades hadrônicas diretamente da QCD. O desenvolvimento da teoria de gauge na rede proporcionou 
alguns sucessos neste sentido [21], mas ainda com resultados pouco satisfatórios.

Sendo assim, a maneira mais direta de se elaborar um modelo para a dinâmica de cor, até aqui não exatamente conhecida, veio com a utilização de modelos fenomenológicos de quarks e glúons. Os efeitos relativísticos em quarks passaram a ser tratados (e assim permanecem) de diversas maneiras: equação de Klein-Gordon [22], equação de Dirac [23], equação de Bethe-Salpeter [24], resolução de equação ou teoria perturbativa no cone de luz $[25,26]$, etc. A equação de Fermi-Yang tem sido usada na resolução de mésons do tipo átomo (quark pesado e antiquark leve) em modelos semi-relativísticos.

Há uma grande variedade de modelos a quarks nãorelativísticos que em geral, permitem a extração das interações de confinamento e residuais a partir da espectroscopia de massas. De Rújula, Georgi e Glashow [27] propuseram a interação quark-quark baseada na troca de um glúon sugerida pela QCD. Partindo-se da equação de Breit [29, 30], com a idéia de que a constante de acoplamento que governa a interação quark-glúon é suficientemente pequena, a interação quark-quark deveria ter uma certa semelhana com a interação elétron-elétron pela troca de um fóton (QED).

Os quarks nos hádrons possuem duas propriedades: a Liberdade Assintótica [13, 14] e o Confinamento ou "Vínculo Infravermelho", parte opostamente correspondente liberdade assintótica para grandes distâncias. Com estas propriedades temos a interação confinante entre quarks em um estado ligado:

$$
U=U_{\text {confinamento }}+U_{\text {troca de um glúon }} \text {. }
$$

Como veremos, o potencial de confinamento, que não decorre diretamente de uma teoria quântica de campos, é fenomenologicamente determinado. O potencial Coulombiano + linear foi proposto primeiramente pelo grupo de Cornell e proporcionou resultados muito bons para o cálculo da espectroscopia de massas do charmônio e do bottomônio [30]. Potenciais logarítmicos e de outras potências também alcançaram relativo sucesso.

Antes de prosseguirmos com o estudo das interações entre quarks fazemos um adendo sobre as massas dos quarks. Como os quarks não são detectados isoladamente, suas massas não podem ser medidas experimentalmente. $\mathrm{Na}$ construção e execução dos modelos de interação podemos optar pelas massas de correntes como as usadas por Bogoliubov [28] no "Bag Model", ou pelas massas constituintes, obtidas da análise dos momentos magnéticos dos bárions [31], adequadas aos modelos de quarks constituintes. A massa constituinte é igual à massa de corrente somada à massa dinâmica [32]. Em mésons do tipo átomo $(Q \bar{q})$, que são aqueles com um quark pesado e um antiquark comparativamente leve, a massa de corrente é mais adequada para o antiquark leve. De qualquer forma, em linhas gerais, utiliza-se a expressão de que as massas dos quarks variam conforme o modelo [30], já que não sabemos como estimar o efeito nãoperturbativo de confinamento da QCD, sendo que os valores das massas estão analiticamente ligados aos parâmetros da Hamiltoniana em questão.
Apesar de particularidades como estas, os modelos de potenciais não-relativísticos têm fornecido resultados extremamente satisfatórios na descrição das propriedades dos quarkônios pesados como também na descrição de hádrons ordinários. Considerações teóricas e aplicações fenomenológicas se misturam de maneira a dificultar o entendimento do que realmente é conseqüência exata das condições teóricas e do que depende dos parâmetros experimentais.

Na seção II fazemos as considerações mais importantes na construção das interações entre quarks dentro do contexto de um modelo de potencial.

$\mathrm{Na}$ seção III obtemos a estrutura de spins conforme a redução não-relativística.

A seção IV é dedicada à análise dos aspectos mais importantes na determinação de um potencial de interação entre duas partículas, no caso específico de um estado ligado quark-antiquark. Essa seção é complementada pela seção $\mathrm{V}$ onde fazemos uma descrição do que pode ser uma forma particular para o potencial de interação quark-antiquark.

Evitamos nos adentrar em qualquer discussão sobre como deve ser feito o tratamento do termo cinético presente na Hamiltoniana efetiva. Existem diversos trabalhos [28-39] que sugerem diferentes abordagens para o tratamento, relativístico ou semi-relativísitico, do termo cinético. Esse tratamento deve ser minuciosamente cuidadoso quando se deseja aplicar um modelo de potencial na obtenção de propriedades físicas de estados ligados, notoriamente, o espectro de massas e as taxas de decaimento.

Na seção VI fazemos algumas observações sobre uma possível metodologia para os cálculos de espectros de massa bem como a viabilidade física e numérica para tal procedimento. Por fim, encerramos este texto com as conclusões na seção VII.

\section{A interação entre quarks e os mo- delos de potenciais}

Ao falarmos de forças entre quarks, correções dependentes de spin, etc, estamos utilizando a linguagem dos modelos de potenciais. Os modelos de potenciais são considerados, teoricamente, menos fundamentados do que os cálculos na rede, as regras de soma da QCD, ou mesmo, o "Bag Model". As duas principais objeções à aplicação dos modelos de potenciais decorrem do efeito do vácuo não trivial da QCD e da não existência de um potencial local implicando na falta de uma justificativa para a aplicação de uma aproximação nãorelativística. Sendo assim, o sucesso dos modelos de potenciais para hádrons ordinários chega a parecer um milagre. Entretanto, com o aprimoramento dos métodos perturbativos, muitas ambigüidades e discussões que se apresentavam foram esclarecidas, resultando em um quadro consistente da QCD [30, 39] sob a conotação dos modelos de potenciais.

O ponto fraco de qualquer modelo sobre a interação entre quarks está justamente na falta de um critério em que se fundamente a validade do mesmo. Nos cálculos na rede, a dimensão mínima do parâmetro de rede, a posição e extensão do comprimento de escala, etc, são determinados empiricamente. Nas regras de soma deve-se escolher um con- 
junto truncado de operadores que testem a estabilidade de complicadas transformações de integrais. E como não poderia ser diferente, os modelos de potenciais devem controlar a validade das aproximações utilizadas, checando sua real aplicabilidade. Isso também é verdade para a teoria de gauge na rede e para as regras de soma.

Em três aspectos os modelos de potenciais são superiores aos demais citados. Através dos mesmos é possível o estudo de todo o espectro de energia dos estados ligados entre quarks. A separação por termos dependentes de spin pode ser facilmente obtida pelo cálculo dos elementos de matriz de uma Hamiltoniana dependente de spin, ao passo que nos demais modelos cada estado ligado exige uma abordagem particular. E finalmente, os modelos de potenciais são bastante claros na interpretação física do fenômeno que ocorre na interação, tratando-se, portanto, de uma ótima referência quantitativa e qualitativa para a descrição das propriedades dos estados ligados.

Um trabalho pioneiro que propôs um modelo de potencial para quarkônios motivado pela QCD foi o modelo de De Rújula, Georgi e Glashow [27].

Desde então foram traçadas as prerrogativas fenomenológicas que caracterizam os modelos de potenciais:

- Os hádrons encontram-se em um singleto de cor formado de quarks "coloridos".

- A curtas distâncias, as interações em QCD obedecem à liberdade assintótica, ou seja, temos uma aproximação para uma teoria não interagente para energias (de interação) elevadas (constante de acoplamento pequena). As interações mediante troca de um glúon tornam-se dominantes e o potencial será preponderantemente Coulombiano (situação bastante similar ao limite quase estático da QED com troca de um fóton).

- A longas distâncias, os quarks estão ligados por um potencial de longo alcance independente de sabor.

- O movimento de um quark no interior de um hádron é descrito efetivamente pela dinâmica relativística com correções relativísticas da ordem de $\frac{v^{2}}{c^{2}}$, as quais são análogas às interações de Breit-Fermi na QED [45].

Sendo assim, em modelos não-relativísticos o potencial motivado pela QCD freqüentemente tem a forma de um termo de troca de um glúon (Coulombiano) e de um termo de confinamento. Como vimos na seção anterior, a principal diferença em relação à interação eletromagnética é a natureza não-abeliana dos glúons, que carregam carga de cor, diferentemente dos fótons, que não têm carga elétrica.

Como o confinamento não é obtido analiticamente de uma teoria quântica de campos, o termo associado é obtido fenomenologicamente com subsídios teóricos indicados pela QCD. Sendo assim, procura-se descrever as interações entre quarks através de uma Hamiltoniana motivado pela QCD.

Em princípio, a descrição da interação dentro de uma abordagem de uma teoria quântica de campos envolve o formalismo de Bethe-Salpeter [46] que, entretanto, sob certos aspectos, apresenta alguns problemas como:

- Em geral, a equação de Bethe-Salpeter não é resolvida analiticamente.

- O elemento de interação não é decorrente dos resultados da QCD.

- Os propagadores dos constituintes são aproximados pela forma não-interagente (propagadores livres), apesar das massas envolvidas serem interpretadas como efetivas.

Há uma enorme dificuldade em resolvermos a equação de Bethe-Salpeter (2) exatamente, devido à falta de conhecimento sobre a energia relativa $k^{0}$ e devido à incompatibilidade com o potencial estático inicial por sua natureza covariante:

$$
\left(\gamma_{\mu} p_{1}{ }^{\mu}-m_{1}\right)\left(\gamma_{\mu} p_{2}{ }^{\mu}-m_{2}\right) \tilde{\psi}(p, P)=\frac{i}{(2 \pi)^{4}} \int U\left(p-p^{\prime}\right) \psi\left(p^{\prime}, P\right) d^{4} p^{\prime}
$$

$\operatorname{com} P=\frac{p_{1}+p_{2}}{2}$ e $p=\frac{m_{1} p_{1}-m_{2} p_{2}}{m_{1}+m_{2}}$.

Em algumas situações opta-se por negligenciar o valor da energia relativa $k^{0}$ fazendo uma aproximação instantânea de maneira que se obtenha a equação de Salpeter [47]:

$$
\left(E-H_{1}-H_{2}\right) \tilde{\phi}(\mathbf{p}, P)=\int d^{3} p^{\prime}\left\{\left[\Lambda_{1}^{+} \gamma_{1}{ }^{0} U\left(\mathbf{p}-\mathbf{p}^{\prime}\right) \tilde{\phi}\left(\mathbf{p}^{\prime}, P\right) \gamma_{2}^{0} \Lambda_{2}^{+}\right]-\left[\Lambda_{1}^{-} \gamma_{1}{ }^{0} U\left(\mathbf{p}-\mathbf{p}^{\prime}\right) \tilde{\phi}\left(\mathbf{p}^{\prime}, P\right) \gamma_{2}^{0} \Lambda_{2}{ }^{-}\right]\right\}
$$

Nesta expressão, $H_{i}$ é a Hamiltoniana de Dirac de uma partícula $i$ do estado ligado. Ainda temos:

$$
\begin{gathered}
\Lambda_{i}^{ \pm}=\frac{1}{2}\left(1 \pm \frac{H_{i}}{\sqrt{p_{i}^{2}+m_{i}^{2}}}\right), \\
\tilde{\phi}(\mathbf{p}, P)=\int d p^{0} \tilde{\psi}\left(p^{0}, \mathbf{p}, P\right) .
\end{gathered}
$$

Esta é uma equação para função de onda em tempo igual com cinemática relativística mas com aproximação estática do potencial. Mantendo a cinemática relativística na equação de Bethe-Salpeter provavelmente chegaríamos a melhores resultados, mas o tratamento pode apresentar algumas complicações na sistemática de cálculos.

A alternativa menos complexa consiste em partirmos para o formalismo de Schrödinger que, na verdade, consiste da aproximação não-relativística aplicada sobre (3). Esta aproximação consiste basicamente em dois procedimentos: tomar o termo cinético segundo uma aproximação não-relativística e fazer uma aproximação local para o po- 
tencial de interação, ambos, no referencial do centro de momento $(P=0)$ :

$$
\begin{gathered}
H_{i}(\text { Dirac }) \longrightarrow T_{i}^{c i n} \\
\Lambda_{i}{ }^{+} U\left(\mathbf{p}-\mathbf{p}^{\prime}\right) \tilde{\phi}\left(\mathbf{p}^{\prime}\right) \Lambda_{i}{ }^{+} \longrightarrow U\left(\mathbf{p}-\mathbf{p}^{\prime}\right) \phi\left(\mathbf{p}^{\prime}\right), \\
\Lambda_{i}{ }^{-} U\left(\mathbf{p}-\mathbf{p}^{\prime}\right) \tilde{\phi}\left(\mathbf{p}^{\prime}\right) \Lambda_{i}{ }^{-} \longrightarrow 0
\end{gathered}
$$

Com esta aproximação temos exatamente a equação de Schrödinger (9):

$$
\left(T_{1+2}^{c i n}-E\right) \tilde{\phi}\left(\mathbf{p}^{\prime}\right)=-\int \frac{d^{3} p^{\prime}}{(2 \pi)^{3}} \tilde{U}\left(\mathbf{p}-\mathbf{p}^{\prime}\right) \tilde{\phi}\left(\mathbf{p}^{\prime}\right),
$$

com:

$$
U(\mathbf{r})=\int \frac{d^{3} k}{(2 \pi)^{3}} \exp (i \mathbf{r} \cdot \mathbf{k}) \tilde{U}(\mathbf{k})
$$

$\mathrm{e} \mathbf{k}=\mathbf{p}-\mathbf{p}^{\prime}$

Como salietamos no início, não nos estenderemos sobre qualquer discussão de como pode ser feita a aproximação não-relativística para o termo cinético.

Considerando o espalhamento elástico dos constituintes de um estado ligado, a parte perturbativamente acessível do potencial pode ser obtida da seguinte maneira:

Escrevemos a amplitude de espalhamento $T_{f i}$ em função dos elementos de menor ordem da matriz $\mathbf{S}$ :

$$
S_{f i}=\langle f \mid i\rangle=\delta_{f i}+i(2 \pi)^{4} \delta^{4}\left(p_{f}-p_{i}\right) T_{f i} .
$$

Para a obtenção do potencial de interação tomamos o limite não-relativístico de $(12)^{1}$ :

$$
\begin{gathered}
U(x)=-\frac{1}{(2 \pi)^{4}} \int d^{4} k\left[\exp (-i k \cdot x) T_{f i}(k)\right] . \\
T_{f i}(k) \longrightarrow-\tilde{U}(\mathbf{k})(2 \pi) \delta\left(k^{0}\right) .
\end{gathered}
$$

A situação relevante no cálculo das forças quarkantiquark $(q \bar{q})$ em um méson vem justamente da análise do espalhamento $q \bar{q}$ :

$$
q_{1}\left(p_{1}, s_{1}\right)+\bar{q}_{2}\left(p_{2}, s_{2}\right) \longrightarrow q_{3}\left(p_{3}, s_{3}\right)+\bar{q}_{4}\left(p_{4}, s_{4}\right) .
$$

O par quark-antiquark em um méson se dispõe em um singleto de cor. A análise dos diagramas de Feynman, considerando apenas os termos em primeira ordem, como pode ser observado em qualquer livro de teoria quântica de campos [32], propicia a obtenção da amplitude de espalhamento no limite não-relativístico:

$$
T_{f i}=\frac{1}{(2 \pi)^{6}} \frac{4}{3} \frac{g_{s}^{2}}{\mathbf{k}^{2}}
$$

onde $g_{s}$ é a constante de acoplamento para interações cromodinâmicas.

Conseqüentemente, a interação quark-antiquark $q \bar{q}$ vista segundo a aproximação em primeira ordem pela troca de um glúon leva a um potencial da forma:

$$
U(r)=-\frac{4}{3} \frac{\alpha_{s}}{r} \quad \text { com } \quad \alpha_{s}=\frac{g_{s}{ }^{2}}{4 \pi} .
$$

No caso de bárions, as forças são determinadas pelo espalhamento quark-quark $(q q)$. No caso em que temos apenas a interação entre dois corpos em um sistema de três corpos o fator de cor será de $-\frac{2}{3}$ e no caso em que temos a interação entre três corpos mediante uma "glueball" de vértice triplo, o fator de cor será nulo [31].

\section{A estrutura de spins e as correções relativísticas}

Procuraremos, agora, uma maneira sistemática de obter as correções de ordem $\frac{1}{m^{2}}$ da equação de Schrödinger nãorelativística, de maneira a observarmos imediatamente a separação do espectro de massas devido aos termos dependentes de spin. As informações sobre os termos dependentes de spin podem permitir um entendimento profundo da natureza (Lorentz) do confinamento.

Em princípio, a determinação das correções relativísticas parece imediata: expandem-se os termos até a ordem de $\frac{1}{m^{2}}$ e identifica-se a Hamiltoniana efetiva que aparece na equação de Schrödinger. Entretanto, um pequeno problema aparece na associação entre os spinores de quatro componentes da amplitude de Bethe-Salpeter e os spinores de duas componentes que aparecem na equação de Schrödinger. Um certo cuidado é necessário de forma que se preserve a normalização correta na passagem da descrição relativística para a não-relativística [30, 48].

A respeito das interações, se tomarmos um kernel $\tilde{I}$ generalizado ao invés do kernel Coulombiano, podemos escrevê-lo como uma composição dos invariantes de Lorentz com paridade definida para cada termo:

$$
\begin{aligned}
\tilde{I}=\tilde{S} 1 \otimes & 1+\tilde{P} \gamma^{5} \otimes \gamma^{5}+\tilde{V} \gamma^{\mu} \otimes \gamma_{\mu} \\
& +\tilde{A} \gamma^{\mu} \gamma^{5} \otimes \gamma_{\mu} \gamma^{5}+\frac{1}{2} \tilde{T} \sigma^{\mu \nu} \otimes \sigma_{\mu \nu}
\end{aligned}
$$

em que estabelecemos que $\tilde{U} \Gamma \otimes \Gamma=\bar{\psi} \Gamma \psi \tilde{U} \bar{\psi} \Gamma \psi$.

As funções $\tilde{S}, \tilde{P}, \tilde{V}, \tilde{A}$ e $\tilde{T}$ são funções escalares que podem depender dos quadrimomentos $p_{\text {in }}(p), p_{\text {out }}\left(p^{\prime}\right)$, $k\left(p-p^{\prime}\right)$ ou $P\left(\frac{p+p^{\prime}}{2}\right)$. No prosseguimento do tratamento, problemas aparecem na integração da componente temporal $p^{0}{ }_{\text {out }}$. A energia transferida entre as partículas interagentes, $k^{0}$, é pequena no limite não-relativístico, portanto, no limite estático podemos adotar $k^{0} \rightarrow 0$.

Uma outra prescrição [49] coloca os quadrimomentos $p_{i}$ de forma que $p_{i}{ }^{0}=\sqrt{m_{i}{ }^{2}+\mathbf{p}_{\mathbf{i}}{ }^{2}}$ [camada de massa ("mass shell")] possibilitando um tratamento mais simples para problemas de espalhamento. Aliás, como já vimos, esta análise com base no formalismo de matriz $\mathbf{S}$ proporciona meios simples de se obter um potencial efetivo: a interação tratada segundo os termos de menor ordem permite que, pelo caminho inverso da primeira aproximação de Born, o potencial seja obtido pela transformada de Fourier da amplitude de espalhamento com relação ao momento transferido. (O mesmo

\footnotetext{
${ }^{1}$ A variável $x$ é o quadrivetor posição, $\mathbf{r}$ é o vetor posição e $r$ é o módulo do vetor posição.
} 
resultado pode ser obtido com uma complicada redução nãorelativística [30] da equação de Bethe-Salpeter sem termos que assumir que a energia de interação é pequena).

$\mathrm{O}$ fato de se colocar as componentes de momento $p_{\text {in }}, p_{\text {out }}, k$ ou $P$ (total) na "camada de massa" ao invés de se adotar a aproximação instantânea, gerou inúmeras ambigüidades, outrora discutidas [45, 49, 50, 51], na maneira de se expressar corretamente a Hamiltoniana efetiva.

Uma sugestão bem mais simples [30] consiste em partirmos de uma teoria perturbativa estacionária do tipo Schrödinger com aproximação estática inicial para, em seguida, considerarmos os desvios desta aproximação.

Outro fato a ficarmos atentos na derivação da Hamiltoniana interagente é no ordenamento correto dos operadores não comutantes que aparecem no potencial efetivo [30]. Para evitarmos maiores problemas com respeito a isso, efetuamos a transformação de Foldy (18) e escrevemos a Hamiltoniana de interação em termos dos momentos $\mathbf{k}$ e $\mathbf{p}$ que são respectivamente o momento de troca entre as partículas e o momento de cada partícula no referencial de centro de momento:

$$
\langle\Gamma\rangle=\psi^{\dagger}\left(\mathbf{p}^{\prime}\right) \Gamma \psi(\mathbf{p})
$$

Impondo a restrição de que a dependência do kernel de interação seja da forma $\tilde{I}=\tilde{I}\left(k^{2}\right)$ e seguindo o procedimento de redução não-relativística, chegaremos ao resultado esperado.

Inúmeros são os trabalhos na tentativa de encontrar uma Hamiltoniana efetiva que descreva com maior precisão os elementos de interação em um estado ligado de dois quarks [33, 36, 39, 45, 49, 50, 51]. Dentro deste contexto, ainda restam controvérsias sobre como expressar exatamente os efeitos de retardamento do potencial. Optamos por tomar como referência a prescrição de Gromes [49] que atenta para os termos dependentes do momento de forma que sejam convenientemente escritos, mantendo-se a hermeticidade da Hamiltoniana.

Utilizando a notação $\beta=\gamma^{0}, \gamma^{0} \alpha=\gamma$ e $\sigma$ representando as matrizes de Pauli (operador de spin $\mathbf{S}=\frac{\sigma}{2}$ ), temos que a função de onda normalizada para o estado livre é escrita como:

$$
u^{s}(\mathbf{p})=\sqrt{\frac{E+m}{2 E}}\left(\begin{array}{c}
\mathbf{1} \\
\frac{\sigma \cdot \mathbf{p}}{E+m}
\end{array}\right) \chi_{s}
$$

onde " 1 " representa a matriz identidade $2 \times 2$.

A redução não-relativística fornece

$$
u^{s}(\mathbf{p})=\left(\begin{array}{c}
\mathbf{1}-\frac{\mathbf{p}^{2}}{8 m^{2}} \\
\frac{\sigma \cdot \mathbf{p}^{2}}{2 m}
\end{array}\right) \chi_{s} .
$$

Segundo as transformações de Foldy, obtemos

$$
\begin{array}{r}
\langle\mathbf{1}\rangle=\mathbf{1}-\frac{\mathbf{k}^{2}}{8 m^{2}}+i \frac{1}{4 m^{2}} \sigma \cdot(\mathbf{k} \times \mathbf{p}), \\
\langle\beta\rangle=\mathbf{1}-\frac{\mathbf{P}^{2}}{2 m^{2}}-i \frac{1}{4 m^{2}} \sigma \cdot(\mathbf{k} \times \mathbf{p}), \\
\langle\alpha\rangle=\frac{\mathbf{P}}{m}-i \frac{1}{2 m}(\sigma \times \mathbf{k}) .
\end{array}
$$

Consideramos somente termos até a ordem de $\frac{1}{\mathrm{~m}^{2}}$.

As funções de $\mathbf{k}$ atuam somente no potencial em questão e as funções de $\mathbf{P}$ (e p) atuam somente na função de onda.

Utilizamos (21) no cálculo dos termos escalar e vetorial presentes na Hamiltoniana efetiva:

\section{A componente escalar $U_{S}(r)$.}

$$
\begin{gathered}
\bar{\psi}_{1} \psi_{1} S(r) \bar{\psi}_{2} \psi_{2} \Rightarrow\left\langle\beta_{1}\right\rangle S(r)\left\langle\beta_{2}\right\rangle \Rightarrow \\
S(r)-\frac{1}{2}\left(\frac{1}{m_{1}^{2}}+\frac{1}{m_{2}^{2}}\right) S(r) \mathbf{P}^{2}-\frac{1}{2}\left(\frac{\mathbf{s}_{\mathbf{1}}}{m_{1}^{2}}+\frac{\mathbf{s}_{\mathbf{2}}}{m_{2}{ }^{2}}\right) \cdot \mathbf{L} \frac{1}{r} \frac{d}{d r} S(r)-\frac{1}{2}\left(\frac{1}{m_{1}{ }^{2}}+\frac{1}{m_{2}{ }^{2}}\right) f\left(\omega_{s}(r)\right),
\end{gathered}
$$

O operador $\mathbf{P}$ é aplicado somente na função de onda e $\omega_{s}(r)$ é a transformada de Fourier de $\frac{d}{d\left(-k^{2}\right)} S\left(-k^{2}\right)$.

Temos $\nabla^{2} \omega_{s}(r)=\frac{1}{2} \nabla \cdot(\mathbf{r} S(r))$ introduzida segundo a já citada prescrição de Gromes [49].

\section{A componente vetorial $U_{V}(r)$.}

- Temporal.

$$
\begin{gathered}
\bar{\psi}_{1} \gamma^{0} \psi_{1} V(r) \bar{\psi}_{2} \gamma^{0} \psi_{2} \Rightarrow\left\langle 1_{1}\right\rangle V(r)\left\langle 1_{2}\right\rangle \Rightarrow \\
V(r)+\frac{1}{8}\left(\frac{1}{m_{1}{ }^{2}}+\frac{1}{m_{2}{ }^{2}}\right) \nabla^{2} S(r)+\frac{1}{2}\left(\frac{\mathbf{s}_{\mathbf{1}}}{m_{1}{ }^{2}}+\frac{\mathbf{s}_{2}}{m_{2}{ }^{2}}\right) \cdot \mathbf{L} \frac{1}{r} \frac{d}{d r} V(r) .
\end{gathered}
$$

- Espacial.

$$
\begin{gathered}
-\left(\bar{\psi}_{1} \gamma \psi_{1} V(r) \bar{\psi}_{2} \gamma \psi_{2}+\bar{\psi}_{1} \gamma \cdot \mathbf{k} \psi_{1} \frac{V(r)}{\mathbf{k}^{2}} \bar{\psi}_{2} \gamma \cdot \mathbf{k} \psi_{2}\right) \Rightarrow \\
\Rightarrow-\left\langle\alpha_{1}\right\rangle V(r)\left\langle\alpha_{2}\right\rangle+\left(\left\langle\alpha_{1}\right\rangle \cdot \mathbf{k}\right) \frac{V(r)}{\mathbf{k}^{2}}\left(\mathbf{k} \cdot\left\langle\alpha_{2}\right\rangle\right) \Rightarrow
\end{gathered}
$$




$$
\frac{1}{m_{1} m_{2}} V(r) \mathbf{P}^{2}+\frac{2 \mathbf{s}_{1} \cdot \mathbf{s}_{2}}{3 m_{1} m_{2}} \nabla^{2} V(r)-\frac{S_{12}}{12 m_{1} m_{2}}\left(\frac{d^{2}}{d r^{2}}-\frac{1}{r} \frac{d}{d r}\right) V(r)+\frac{1}{m_{1} m_{2}} \mathbf{s} \cdot \mathbf{L} \frac{1}{r} \frac{d}{d r} V(r)+\frac{1}{m_{1} m_{2}} f(\omega(r)) .
$$

Novamente o operador $\mathbf{P}$ atua somente na função de onda. Temos ainda que

$$
\begin{gathered}
S_{12}=3\left(\mathbf{s}_{1} \cdot \hat{n}\right)\left(\mathbf{s}_{2} \cdot \hat{n}\right)-\left(\mathbf{s}_{1} \cdot \mathbf{s}_{2}\right), \\
f(\omega(r))=(\mathbf{P} \cdot \nabla) \omega(r) .
\end{gathered}
$$

Da mesma maneira $\omega(r)$ é a transformada de Fourier de $\frac{d}{d\left(-k^{2}\right)} V\left(-k^{2}\right)$ satisfazendo $\nabla^{2} \omega(r)=-V(r)$.

Os demais termos: pseudo-escalar, axial (ou pseudo-vetorial) e tensorial, também são calculados segundo o mesmo procedimento:

A componente pseudo-escalar $U_{P}(r)$.

$$
\frac{1}{3 m_{1} m_{2}} \nabla^{2} P(r) \mathbf{s}_{1} \cdot \mathbf{s}_{2}+\frac{2 S_{12}}{3 m_{1} m_{2}}\left(\frac{d^{2}}{d r^{2}}-\frac{1}{r} \frac{d}{d r}\right) P(r)
$$

A componente axial $U_{A}(r)$.

$$
-4 A(r) \mathbf{s}_{1} \cdot \mathbf{s}_{2}+O\left(\frac{1}{m^{2}}\right)
$$

\section{A componente tensorial $U_{T}(r)$.}

$$
+4 T(r) \mathbf{s}_{1} \cdot \mathbf{s}_{2}+O\left(\frac{1}{m^{2}}\right)
$$

As formas explícitas das correções axial e tensorial não são fisicamente muito interessantes (na interação quark-antiquark não temos propagadores com essas características). Uma interação pseudo-escalar tem sua importância em alguns modelos nucleares com interação via propagadores pseudo-escalares (troca de um píon na ligação do dêuteron por exemplo).

De qualquer forma, é importante observar que somente as componentes escalar e vetorial apresentam termos puros de potencial: $S(r)$ e $V(r)$. As prerrogativas físicas impõem na Hamiltoniana efetiva de interação somente a presença das componentes vetorial e escalar para os sistemas quarkantiquark. É importante salientar que essa Hamiltoniana contém todos os termos de interação de Breit-Fermi.

Os termos da Hamiltoniana que vêm da interação escalar são bem mais simples, com apenas um termo de interação dependente de spin: spin-órbita (pode ser interpretado como o termo de precessão de Thomas).

Dos resultados teóricos e fenomenológicos [30] que temos até agora, há uma série de argumentos muito convincentes de que as forças entre quarks vêm da combinação de um termo Coulombiano de troca vetorial de curto alcance (um glúon) e um termo linear de confinamento escalar. O sinal oposto entre os termos de interação spin-órbita vetorial e escalar leva a uma compensação de tais efeitos, ou seja, sem esta característica a separação de energias devido a esses termos não comportaria um tratamento perturbativo.
É importante constar que a determinação da estrutura de Lorentz do potencial de interação Coulombiano + linear decorre de uma análise simplificada dos dados experimentais [30]. Diante disso, alguns autores [34, 35, 36] optam por parametrizar este comportamento, ao invés da denominação vetorial para o termo Coulombiano e escalar para o termo linear. Algo desse tipo é feito quando fazemos os cálculos com o potencial logarítmico, mas de qualquer forma, tal procedimento não acrescenta nenhum conteúdo físico ao problema.

Voltando a falar do potencial pseudo-escalar, observamos a ausência de um potencial estático e a presença de um termo spin-spin e um termo tensorial que não promovem uma interação muito forte.

Com relação à existência de propagadores axiais ou tensoriais, teríamos as interações spin-spin bem mais significativas $\left(O\left(1>>\frac{1}{m^{2}}\right)\right)$, o que definitivamente não observamos na espectroscopia de energia que temos experimentalmente.

\section{Aspectos gerais do potencial de interação entre quarks}

De acordo com a análise da estrutura de spins do potencial, os indícios fenomenológicos mostram que o potencial da interação quark-antiquark deve conter uma componente es- 
calar e uma vetorial $[30,52,53]$. Para pequenas distâncias, onde a interação é mediada pela troca de um glúon de alta energia, o potencial do tipo Coulombiano ponderado por um fator de cor constitui uma boa aproximação em primeira ordem. Para grandes distâncias deve haver uma contribuição que descreva o confinamento de cor. Dinamicamente, diretamente da QCD, não temos uma expressão analítica que descreva uma interação pela troca de um quantum de campo associado à força confinante. Fenomenologicamente, supõese

$$
U(r)_{\text {confinamento }}=a r^{n}, \quad n>0 .
$$

Os dados experimentais indicam que $n$ é aproximadamente igual a um, assim, o potencial completo ficaria dado por

$$
U(r)=-\frac{4 \alpha_{s}}{3 r}+a r+b .
$$

A origem do termo constante $b$ vem da divergência infravermelha da integral de Fourier (36) do kernel de confinamento que leva ao potencial de confinamento (o valor de $b$ depende dos parâmetros de confinamento e da escala de momento $\rightarrow$ ponto de renormalização, mas para todos os efeitos é determinado fenomenologicamente)

$$
\int d^{3} k \frac{\exp (-i \mathbf{k} \cdot \mathbf{r})}{\mathbf{k}^{4}} \approx r
$$

Potenciais da forma (35) têm o termo Coulombiano com caráter vetorial e o termo linear com caráter escalar [30].

Alguns modelos limitam a ação destes potenciais para um intervalo de energia de troca (momento transversal) específico: os estados ligados de quarks leves teriam o termo linear de interação predominante e os estados ligados de quarks pesados (bottomônio e topônio) seriam muito bem descritos pelo termo Coulombiano dominante. Nessa linha, alguns modelos com potencial logarítmico, correspondente justamente à região intermediária de (35), são muito bem aplicados no estudo do charmônio e do bottomônio [38].

Uma forma simples de se interpolar as regiões de ação desses potenciais é através do potencial de Richardson [30], cuja característica é depender de um único parâmetro $\Lambda$ :

$$
U(r)=-\frac{4}{3 B} \int \frac{d^{3} k}{(2 \pi)^{3}} \frac{\exp (-i \mathbf{k} \cdot \mathbf{r})}{\mathbf{k}^{2} \ln \left(1+\frac{\mathbf{k}^{2}}{\Lambda^{2}}\right)},
$$

com $B=\frac{33-n_{f}}{48 \pi^{2}}$, em que $n_{f}$ é o número de famílias de quarks em questão (três).

Não é possível fornecer uma expressão analítica para este potencial, mas para seus extremos encontramos

$$
\begin{aligned}
& \mathbf{k}^{2}>>\Lambda^{2} \Rightarrow \\
& \frac{1}{\mathbf{k}^{2} \ln \left(1+\frac{\mathbf{k}^{2}}{\Lambda^{2}}\right)} \approx \frac{1}{\mathbf{k}^{2} \ln \left(\frac{\mathbf{k}^{2}}{\Lambda^{2}}\right)}=\frac{4 \pi B \alpha_{s}\left(\mathbf{k}^{2}\right)}{\mathbf{k}^{2}} \\
& \mathbf{k}^{2}<<\Lambda^{2} \Rightarrow \\
& \frac{1}{\mathbf{k}^{2} \ln \left(1+\frac{\mathbf{k}^{2}}{\Lambda^{2}}\right)} \approx \frac{\Lambda^{2}}{\mathbf{k}^{4}}
\end{aligned}
$$

Para distâncias curtas este potencial apresenta um comportamento linear e para distâncias longas é exatamente igual à aproximação pela troca de um glúon, que no caso de $\alpha_{s}(\mathbf{k}) \rightarrow \alpha_{s}$ equivale ao potencial do tipo Coulombiano. Entretanto, não vemos conveniência na parametrização em termos de um único parâmetro pois não temos nenhum indício de que existe esta correlação entre os dois comportamentos para distâncias longas e curtas (ainda precisa ser verificado fenomenologicamente).

Em resumo, os potenciais que se apresentam são variações do potencial Coulombiano + linear que num tratamento perturbativo aparecem como potenciais estáticos somados às correções de Breit-Fermi com termos relativísticos até $2^{a}$ ordem do inverso das massas constituintes dos quarks nos estados ligados.

De maneira a se obter os autoestados, a equação de Schrödinger pode ser resolvida numericamente sem os termos perturbativos. $\mathrm{O}$ espectro de energia do sistema em consideração é obtido aproximadamente pelos valores esperados da Hamiltoniana completa, calculado em função dos estados não-perturbados. Não há diferença entre os autoestados de spins diferentes. Em princípio, estes modelos são designados para sistemas de quarks pesados.

Para sistemas de estados ligados constituídos de quarks leves o problema é mais difícil, pois o tratamento perturbativo não pode ser justificado. Um dos fatores é a diferença entre o singleto e o tripleto de spin de um mesmo estado cuja diferença de massa é muito elevada (mésons $\pi$ e $\rho$ por exemplo), sendo da ordem ou maior que as massas constituintes do estado ligado.

A interação spin-spin que causa esta discrepância não pode ser tratada perturbativamente.

Conseqüentemente, para quarks leves, as correções da Hamiltoniana de Breit-Fermi precisam ser tratadas nãoperturbativamente, ou seja, a equação de Schrödinger deve ser resolvida com todos os termos da Hamiltoniana.

Para um potencial com termo Coulombiano, a inclusão dos termos relativísticos pode causar problemas de singularidade, do tipo

$$
\nabla^{2}\left(\frac{1}{r}\right)=-4 \pi \delta^{3}(\mathbf{r})
$$

Para uma Hamiltoniana da forma $H=T^{c i n}+H_{s s}$, que apresente o termo acima explicitamente, com a aplicação do método variacional, esta expressão não teria limitante inferior $[30,39,33,35]$ o que significa que não teríamos um estado fundamental, sendo portanto, fisicamente inconcebível. A função delta é conseqüência da aproximação relativística para um modelo de potencial. Este problema pode ser contornado ao deixarmos para tomar o limite não-relativístico para a interação spin-spin somente na última etapa.

A solução mais simples imaginada é o "smearing" da função delta substituindo-a por uma função que converge fracamente para $\delta^{3}(\mathbf{r})$ :

$$
\delta^{3}(\mathbf{r})=\frac{1}{4 \pi r_{0}^{2}} \frac{\exp \left(-\frac{r}{r_{0}}\right)}{r}
$$


Este procedimento pode ser aplicado ao potencial Coulombiano + linear acrescido de um termo exponencial [30].

Outra possibilidade é introduzir um termo de delocalização na parte Coulombiana do potencial na forma

$$
\begin{aligned}
& \operatorname{erf}(r)=\frac{2}{\sqrt{\pi}} \int_{0}^{r} \exp \left(-t^{2}\right) \Rightarrow \\
& \nabla^{2}\left(\frac{\operatorname{erf}(r)}{r}\right)=\frac{4}{\sqrt{\pi}} \exp \left(-r^{2}\right) .
\end{aligned}
$$

Além de todos os aspectos discutidos nesta seção, é importante ressaltar o caráter da função de onda na origem em um termo de aniquilação na Hamiltoniana de interação [33, 35]. Em mésons, a aniquilação por um único glúon é proibida por conservação de cor, mas a aniquilação via vários glúons pode ser esperada. Para estados de quarks pesados, onde o processo de aniquilação é controlado (probabilidade) pela constante de acoplamento suficientemente pequena, o processo é dominado por um número mínimo de glúons ( 2 ou 3 , conforme a conjugação de cargas). Godfrey e Isgur [33] sugerem uma contribuição à Hamiltoniana proporcional a

$$
\alpha_{s}{ }^{n} \frac{\left|\psi_{q \bar{q}}\right|}{M_{q}^{2}}
$$

não nula apenas para $L=0$ ( $n$ é o número de glúons).

Podemos notar ainda, nesta aproximação, que somente para o estado de singleto um termo desta forma adquire uma dimensão significativa, sendo, para o estado de tripleto, bem pequeno se comparado aos termos de estrutura hiperfina. Portanto, para sistemas de quarks pesados, o único estado para o qual os cálculos poderão divergir em virtude da ausência explícita deste termo na Hamiltoniana [33] seria o estado $1^{1} S_{0}$. Para estados ligados de quarks leves, na totalidade dos trabalhos que analisamos, com destaque para [33-36], o termo de aniquilação assume uma conotação fenomenológica em forma de mais um parâmetro a ser determinado. Ainda há uma discussão intensa de como esses efeitos devem ser implementados, uma vez que qualquer que seja a descrição, mesmo para mésons leves, esta deve assumir valores médios muito pequenos diante das incertezas dos cálculos e mesmo das incertezas experimentais [33].

\section{Uma forma particular para o po- tencial de interação entre quarks}

Para que um potencial na forma Coulombiana + linear seja implementado no desenvolvimento de um modelo fenomenológico, algumas observações são necessárias. Consideramos um caso particular na forma como essa implementação pode ser feita.

Sabemos que, em uma aproximação até primeira ordem dos diagramas de Feynman, a QCD fornece uma expressão para a constante de acoplamento [54] na forma de:

$$
\alpha_{s}\left(k^{2}\right)=\frac{12 \pi}{\left(33-2 n_{f}\right) \ln \left(\frac{k^{2}}{\Lambda^{2}}\right)} .
$$

O parâmetro de escala $\Lambda$ é dado no intervalo de $0,15<$ $\Lambda<0,30 \mathrm{GeV}$, para $n_{f}=4$ ou 5 [54]. Isto indica, conforme (44), que $\alpha_{s}\left(k^{2}\right)$ tende a um valor aproximadamente constante para a região $5<k<20 \mathrm{GeV}$. Por outro lado, quando $k \longrightarrow \Lambda$, o tratamento perturbativo em primeira ordem não se aplica pois (44) diverge indicando a existência do confinamento.

Godfrey e Isgur [33] propõem a parametrização deste comportamento para a região $5<k<20 \mathrm{GeV}$ da seguinte maneira:

$$
\begin{gathered}
\alpha_{s}\left(k^{2}\right)=\sum_{m} \alpha_{m} \exp \left(-\frac{k^{2}}{\eta_{m}^{2}}\right) \Rightarrow \\
\Rightarrow \alpha_{s}(r)=\frac{2}{\sqrt{\pi}} \sum_{m} \alpha_{m} \int_{0}^{\frac{\mathrm{w}_{m} r}{2}} \exp \left(-x^{2}\right) d x . \\
\alpha_{s}(r) \approx \alpha_{s}(1-\exp (-\mathrm{w} r)) .
\end{gathered}
$$

Esta parametrização é procedente uma vez que ela acaba por descrever com bastante propriedade o comportamento assintótico da constante de acoplamento. A partir do momento em que obtemos a constante de acoplamento forte segundo um tratamento perturbativo ( $k^{2}$ elevado), considerando apenas uma expansão diagramática em primeira ordem, é conveniente assumí-la como tal somente para esta região acessível a um tratamento perturbativo.

O confinamento deve aparecer separadamente com os dados experimentais indicando um comportamento linear (47). Na região de interação $5<k<20 \mathrm{GeV}$ apenas o primeiro termo da somatória em (45) é suficiente para parametrizar o comportamento do potencial que propomos (47), e em função de um parâmetro $w$ podemos reproduzir este comportamento dentro da região citada através da parametrização exponencial em (46). Veja na figura 1 como podemos perfeitamente adotar uma parametrização exponencial em $w$ ao invés da expressão em termos da função erro de (45), simplificando os cálculos das autoenergias.

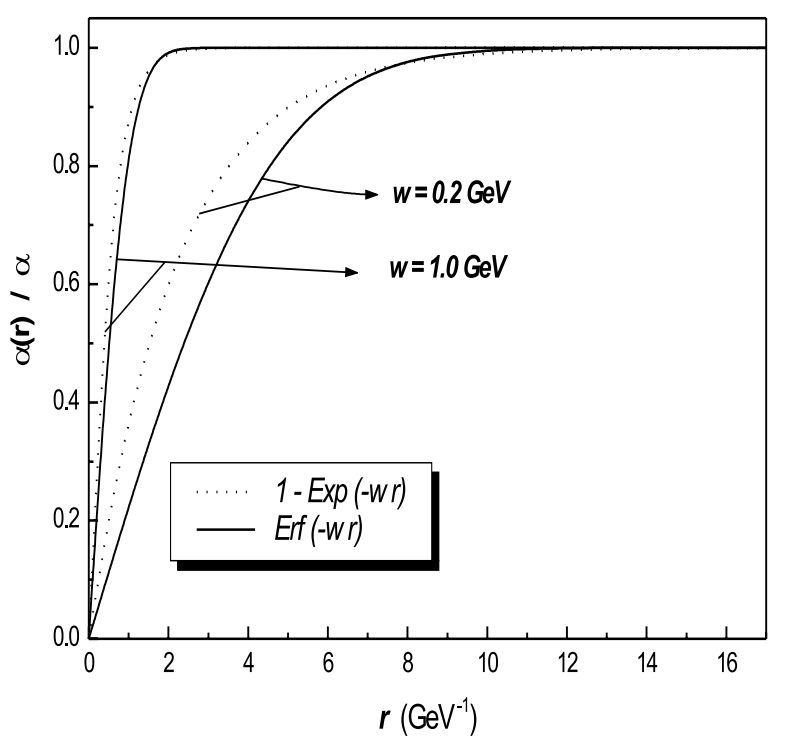

Figura 1. Parametrização da constante de acoplamento do potencial Coulombiano para $w=0.2$ e $w=1.0 \mathrm{GeV}$. 
É importante lembrar que em um estado ligado o quark é tido como um grau de liberdade efetivo "camuflado" por uma nuvem de glúons e pares quark-antiquark (renormalização), de maneira que alguns trabalhos [33, 34, 35] propõem uma densidade de probabilidade de quarks no espaço de configuração como uma função que venha a descrever o efeito de delocalização do potencial. O efeito de um termo como este em uma descrição simplificada como a proposta neste texto, pode ser englobado pelo parâmetro $w$ da constante de acoplamento variável (45).

Além disso, tal parametrização elimina o problema decorrente da ação do operador $\nabla^{2}$ na expressão (40) ${ }^{2}$ :

$$
U(r)=-\frac{4}{3} \frac{\alpha_{s}}{r}(1-\exp (-\mathrm{w} r))+a r+b .
$$

Se pensarmos em um desenvolvimento centrado no estudo dos estados ligados do charmônio e do bottomônio ainda fazemos menção ao potencial logarítmico [38] na forma

$$
U_{l n}(r)=\left(a_{s}+a_{v}\right) r \ln \left(\frac{r}{r_{0}}\right)
$$

em que os parâmetros $a_{s}$ e $a_{v}$ se referem às componentes escalar e vetorial.

\section{Considerações sobre metodologia de cálculos e aplicabilidade}

Nesta seção resumimos o que podemos considerar como algumas diretrizes sugeridas para o cálculo das propriedades físicas, notoriamente o espectro de massas, para grupos distintos de estados ligados quark-antiquark: quarkônios pesados e mésons leves. As sugestões que seguem implícitas apenas complementam as idéias fundamentais que orientam a construção de um modelo de potencial dentro de uma teoria perturbativa com redução não-relativística.

Buscando uma maior simplicidade nos cálculos, em detrimento de métodos mais complicados como o método matricial de colocação ortogonal $[43,44]$ ou de um método variacional que não proporcionaria um resultado tão exato $[34,35,36]$, podemos optar ${ }^{3}$ pela simplicidade de adotarmos uma minimização dos erros quadráticos médios das massas de um número fixo de ressonâncias de alguns estados ligados que em função da precisão de seus valores detectados experimentalmente possam se mostrar como excelentes parâmetros de entrada.

Para a função de onda na forma $\psi(\mathbf{r})=R_{n l}(r) Y_{l m}(\hat{r})$, podemos escrever a parte radial da equação de onda para um estado ligado quark-antiquark como

$$
\left(T^{c i n}+U_{s}(r)+U_{v}(r)-E_{n l}\right) R_{n l}(r)=0 .
$$

Os termos $U_{s}(r)$ e $U_{v}(r)$ envolvem todas as correções decorrentes da redução não-relativística. Esta é justamente uma das vantagens do procedimento de cálculo citado, pois permite o levantamento da espectroscopia completa (todos os números quânticos) de um sistema quark-antiquark.

A escolha mais apropriada de uma função radial de base depende em geral do problema a ser resolvido. Esta escolha, em geral, deve ser favorecida pela viabilidade numérica que implicaria em uma menor magnitude dos erros em uma integração numérica.

Em princípio, é possível trabalhar com três situações diferentes: as autofunções de Schrödinger para o potencial Coulombiano, as autofunções de Schrödinger para o potencial de oscilador harmônico 3D e uma função de base proposta por Fulcher [41] A última dentre as três está descrita em (50) e até certo ponto apresenta um comportamento semelhante ao da função de onda de Schrödinger para um potencial Coulombiano, já tendo sido usada em vários trabalhos [40, 41, 43, 44]:

$$
R_{n l}(r)=\sqrt{\frac{(2 \beta)^{3} k !}{(k+2 l+2) !}}(2 \beta r)^{l} e^{(-\beta r)} L_{k}^{2 l+2}(2 \beta r)
$$

em que $L_{k}{ }^{2 l+2}(\beta r)$ é o polinômio associado de Laguerre e o número quântico principal $n$ com o qual estaremos descrevendo os estados ligados $n^{2 s+1} l_{J}$ é dado em função de $k$ por $n=k+1^{4}$.

Para o estudo do charmônio e do bottomônio, os cálculos são desenvolvidos em termos dos seguintes parâmetros: as massas efetivas dos quarks charm $\left(m_{c}\right)$ e bottom $\left(m_{b}\right)$, um parâmetro $\beta$ da função de onda além dos parâmetros do potencial $(\alpha, a, b$ e $\omega)$. Estes parâmetros são determinados segundo uma minimização quadrática média com base na espectroscopia de massas dos estados de momento angular orbital nulo do charmônio e do bottomônio (em um único conjunto de parâmetros, ou seja, teremos os parâmetros do potencial e da função de onda em comum para o charmônio e para o bottomônio). Caso a interação seja descrita pelo potencial logarítmico, podemos mudar os parâmetros do potencial para $a_{s}, a_{v}$ e $r_{0}$.

Ainda com relação aos parâmetros, o parâmetro $w$ da exponencial que parametriza o termo de interação vetorial, a partir de um determinado valor, faz com que o comportamento de tal termo tenda a um termo Coulombiano puro, conforme os níveis de energia que venham a ser tratados ${ }^{5}$.

Estas ferramentas são suficientes e podem ser aplicadas no estudo da espectroscopia de massas do charmônio e bottomônio.

O grande entrave em estendermos a descrição nãorelativística ou semi-relativística de um problema de

\footnotetext{
${ }^{2}$ Obviamente, a expressão (47) descreve apenas os termos puros do potencial $U(r)=S(r)+V(r)$ sem as correções relativísticas.

${ }^{3}$ Chegamos a estudar a viabilidade dos dois métodos citados, mas estes, apesar de eficazes no cálculo do espectro de massas de um conjunto pequeno de estados ressonantes com momento angular orbital fixo, não apresentam a mesma exatidão e muito menos chegam a ser mais simples no caso em que procuramos descrever a espectroscopia completa (todas as possibilidades dos números quânticos) de um méson.

${ }^{4}$ É muito importante o esclarecimento deste ponto pois existe uma grande confusão na literatura sobre o que indica o número quântico principal. Nos diversos "Particle Data Group" (PDG) não há esclarecimentos sobre o que o índice $n$ de diversos estados ligados realmente significa. Escolhemos a definição $n=k+1$ de maneira que o número quântico principal $n$ corresponda à mesma notação do PDG. Em algumas situações a definição $n=k-l+1$ também é cabível.

${ }^{5}$ Tal comportamento é esperado para quarks pesados.
} 
interação entre quarks pesados para um problema de interação entre quarks leves está justamente no fato de que o momento linear intrínseco de cada quark leve assume valores, comparativamente às suas massas, muito mais significativos do que em sistemas de quarks pesados. Isso impõe, diante destes sistemas de quarks leves, a necessidade de um tratamento relativístico que, de certa forma, passa a gerar um número considerável de restrições no que se refere à simplificação ou mesmo à execução dos cálculos das propriedades destes sistemas. Uma das soluções encontradas para contornar esse tipo de problema, segundo uma dinâmica não-relativística, é justamente procurar associar as previsões de uma dinâmica não-relativística com as previsões de uma dinâmica relativística ou até mesmo ultrarelativística $\left(m_{q} \rightarrow 0\right)$, e através desse vínculo, possibilitar a descrição de um estado ligado relativístico segundo uma dinâmica não-relativística.

Buscando a extensão do nosso modelo não-relativístico a esses sistemas, descreveremos a seguir algumas maneiras de se fazer presente o vínculo entre a dinâmica não-relativística e a dinâmica (ultra) relativística.

Seja a Hamiltoniana relativística com potencial de interação $U(r)$ para o caso mais simples de duas partículas de mesma massa no referencial de centro de momento:

$$
H_{R}=2\left(\mathbf{p}^{2}+m^{2}\right)^{\frac{1}{2}}+U(r) .
$$

Utilizando a desigualdade de Schwarz

$$
|\langle A\rangle| \leq \sqrt{\left\langle A^{2}\right\rangle}
$$

obtemos a desigualdade

$$
\left\langle\left(\mathbf{p}^{2}+m^{2}\right)^{\frac{1}{2}}\right\rangle \leq\left(\left\langle\mathbf{p}^{2}\right\rangle+m^{2}\right)^{\frac{1}{2}},
$$

e a relação

$$
\begin{aligned}
E= & \left.\left\langle H_{R}\right\rangle\right\rangle \\
& \leq 2\left(\left\langle\mathbf{p}^{2}\right\rangle+m^{2}\right)^{\frac{1}{2}}+\langle U(r)\rangle= \\
& =2\left\langle\frac{\left(\mathbf{p}^{2}+m^{2}\right)}{\left(\left\langle\mathbf{p}^{2}\right\rangle+m^{2}\right)^{\frac{1}{2}}}+U(r)\right\rangle .
\end{aligned}
$$

Este operador tem estrutura semelhante ao de um potencial não-relativístico como (57) de acordo com as seguintes transformações de variáveis:

$$
\begin{gathered}
\hat{m}=2 \mu=\frac{1}{2}\left(\left\langle\mathbf{p}^{2}\right\rangle+m^{2}\right)^{\frac{1}{2}}+U(r), \\
W(r)=2 \hat{m}-\frac{\left\langle\mathbf{p}^{2}\right\rangle}{\hat{m}}+U(r), \\
H_{N R}=2 \hat{m}+\frac{\mathbf{p}^{2}}{2 \mu}+W(r) .
\end{gathered}
$$

A massa efetiva $\hat{m}$, bem como o potencial $W(r)$, dependem do valor médio do momento, variando de nível para nível de energia. $\mathrm{O}$ valor esperado da energia cinética (teorema do virial [30]) será expresso por

$$
\left\langle\mathbf{p}^{2}\right\rangle \propto\left\langle r \frac{d U(r)}{d r}\right\rangle .
$$

O mais interessante é que o desenvolvimento em termos da desigualdade de Martin, ou propriamente em termos de uma série de Taylor em torno de um valor $p_{0}^{2}=\left\langle\mathbf{p}^{2}\right\rangle$, pode levar às mesmas conclusões [30, 40].

Uma outra maneira de procurarmos encontrar uma equivalência de modelos não-relativísticos para sistemas quark-antiquark relativísticos na região assintótica vem da observação de uma certa dualidade entre uma Hamiltoniana ultra-relativística com um potencial de oscilador harmônico (59) e uma Hamiltoniana não-relativística com potencial linear (60), relacionadas pela transformação dual:

$$
\begin{gathered}
H_{U R}=2\left(\mathbf{p}^{2}\right)^{\frac{1}{2}}+\kappa r^{2} . \\
H_{N R}=2 \frac{\mathbf{p}^{2}}{2 \mu}+a r . \\
|\mathbf{p}| \leftrightarrow \frac{a r}{2} \operatorname{com} \kappa=\frac{a^{2}}{8 \mu} .
\end{gathered}
$$

As autofunções nas equações de onda correspondentes estão relacionadas pela transformada de Fourier a seguir

$$
H_{U R} \Phi(\mathbf{x})=E \Phi(\mathbf{x})
$$

$$
H_{N R} \Psi(\mathbf{y})=E \Psi(\mathbf{y})
$$

$$
\Phi(\mathbf{x})=\int d^{3} y \exp \left(i \frac{a}{2} \mathbf{x} \cdot \mathbf{y}\right) \Psi(\mathbf{y}) .
$$

Ou seja, podemos encontrar o mesmo espectro de massas dos mésons tanto para uma prescrição não-relativística como para uma prescrição ultra-relativística.

Um terceiro ponto de vista a ser adotado na comparação de uma prescrição não-relativística versus uma prescrição ultra-relativística decorre de uma generalização relativística para o teorema do virial [30] com valores médios calculados segundo os autoestados da Hamiltoniana relativística

$$
\left\langle r \frac{d U(r)}{d r}\right\rangle=2\left\langle\frac{\mathbf{p}^{2}}{\left(\mathbf{p}^{2}+m^{2}\right)^{\frac{1}{2}}}\right\rangle .
$$

Obtemos a autoenergia relativística (63)

$$
E=\left\langle H_{R}\right\rangle=2\left\langle\left(\mathbf{p}^{2}+m^{2}\right)^{\frac{1}{2}}\right\rangle+\langle U(r)\rangle .
$$

Do teorema virial expresso por (62) juntamente com o limite ultra-relativístico de (63) encontramos que

$$
E_{U R}=\left\langle r \frac{d U(r)}{d r}\right\rangle+\langle U(r)\rangle
$$

Escolhendo o potencial do tipo Coulombiano + linear teremos o desaparecimento do termo Coulombiano no cálculo de $E_{U R}$ segundo (64)

$$
E_{U R}=\langle 2 a r\rangle
$$

Concluindo, até o ponto em que o valor do termo de massa pode ser negligenciado (que pode ser o caso dos 
mésons de quarks leves u (up) e d (down)), o valor esperado de uma Hamiltoniana não-relativística com potencial puramente linear tem semelhança formal com a Hamiltoniana relativística com potencial Coulombiano + linear. Os autoestados são obviamente diferentes.

Devemos, porém, deixar claro que as considerações em cima do teorema virial descrevem muito bem a interação decorrente unicamente dos termos puros (sem as correções relativísticas) do potencial.

Sendo assim, para um potencial com parametrização Coulombiana + linear, em estados ligados de quarks leves (sistemas aproximadamente ultra-relativísticos), a semelhança acima sugere um caráter de confinamento mais intenso. Esta observação é muito importante uma vez que este comportamento se inverte à medida em que as massas dos quarks constituintes aumentam (charmônio e bottomônio).

Com estas pequenas considerações explanadas até aqui, procuramos chamar a atenção para o fato de que, dentro de um modelo de potencial em sua forma mais simples, a dinâmica real (física) das interações em um sistema quark-antiquark pode ser parametrizada em termos de uma dinâmica mais simples, na maioria das vezes nãorelativística, de forma que a correlação se dê em termos dos parâmetros fenomenológicos que aparecem na construção da Hamiltoniana que pretende descrever o espectro de energias de uma determinada família de mésons.

A diferença de massas muito acentuada de sistemas como os quarkônios para com sistemas de mésons leves impede o desenvolvimento de um modelo unificado para a descrição da espectro de energias em termos de um número reduzido de parâmetros, como tem sido o objetivo da maioria dos trabalhos. Entretanto, a correspondência entre as prescrições discutidas nesta seção, em princípio, possibilita a generalização de uma prescrição com aproximação não-relativística para sistemas de quarks leves, apenas com a mudança dos parâmetros fenomenológicos da Hamiltoniana.

\section{Conclusões}

Neste texto procuramos elaborar e discutir os aspectos mais importantes na construção de um modelo de potencial nãorelativísitico para o estudo das interações quark-antiquark. Direcionamos o texto de modo a elaborar uma descrição generalizada de como podemos obter os elementos que caracterizam o processo de interação em um estado ligado.

Devido a sua importância histórica e funcional no estudo da física hadrônica, um modelo de potencial, em linhas gerais, não se atém ao estudo de estados ligados quarkantiquark. Modelos de potenciais são modelos fenomenológicos cuja aplicabilidade se estende à física nuclear, bem como à física atômica e molecular.

No caso específico da interação quark-antiquark, um modelo de potencial desenvolvido com os subsídios teóricos da cromodinâmica quântica pode ser utilizado na descrição ampla e precisa do espectro de massas de quarkônios pesa- dos e até mesmo de mésons leves.

Efetivamente, no desenvolvimento de um modelo de potencial para interações em sistemas de dois corpos, relativísticos ou não, são vários e igualmente importantes os fatores que determinam o sucesso do modelo: o número de parâmetros fenomenológicos e a maneira como será dado o tratamento numérico do problema, a maneira como se procede a redução não-relativística em alguns modelos e a maneira como são parametrizados os termos de interações nãolocais entre outros.

Há também inúmeras sugestões para o tratamento de equações de onda relativísticas, modelos relativísticos, etc, que também apresentam virtudes e inconsistências. Parece ironia, mas é extremamente improvável que alguém se dê ao enorme trabalho de rever todas as proposições existentes e de fato encontrar alguma isenta de qualquer defeito; seria mais viável propor uma nova equação descrevendo as interações presentes.

Sobre estdados ligados quark-antiquark, esperamos que alguns outros resultados diretos ou indiretos da QCD possam ter maior influência na elaboração dos modelos fenomenológicos de interação. Neste contexto, a base teórica fornecida pela QCD se apresenta de forma bastante sólida, mesmo diante de algumas ambigüidades decorrentes de alguns comportamentos controversos ${ }^{6}$ que aparecem nos cálculos perturbativos, que só poderão ser evitados, ou melhor entendidos, após uma extensa análise dos fundamentos da cromodinâmica quântica, independente do que temos na fenomenologia.

Com todas essa possibilidades, esperamos ter contribuído um pouco para o conhecimento do processo de elaboração de modelos fenomenológicos no estudo das interações entre quarks e, de maneira geral, entre duas partículas em um estado ligado.

\section{Agradecimentos}

Gostaria de agradecer a CAPES, mantenedora financeira dos meus trabalhos no IFGW.

\section{Referências}

[1] E. Fermi, Phys. Rev. 76, 1739 (1949).

[2] M. Gell-Mann, Phys. Rev. 92, 883 (1953).

[3] T. Nakano and K. Nishijima, Phys. Rev. 92, 883 (1953).

[4] S. Sakata, Phys. Rep. 16, 686 (1956).

[5] M. Gell-Mann, Phys. Lett. 8, 214 (1964).

[6] G. Zweig, CERN 8142 Th 401 prepr. , (1964)

[7] G. Zweig, CERN 8419 Th 412 prepr. , (1964)

[8] Uma extensa bibliografia sobre o modelo a quarks é dada por O. W. Greenberg, Am. J. Phys. 50, 1074 (1982).

[9] J. J. Aubert et al, Phys. Rev. Lett. 33, 1404 (1974).

[10] J. E. Augustin et al, Phys. Rev. Lett. 33, 1406 (1974).

[11] S. Weinberg, Phys. Rev. Lett. 31, 494 (1973).

\footnotetext{
${ }^{6}$ Quando se compara a outros modelos generalizados como com QCD na rede, regras de soma, redução de Bethe-Salpeter, etc.
} 
[12] H. Fritzsch, M. Gell-Mann and H. Leutwyler, Phys. Lett. B 47, 365 (1973).

[13] H. D. Politzer, Phys. Rev. Lett. 30, 1346 (1973).

[14] D. J. Gross and F. Wilckzek, Phys. Rev. Lett. 30, 1343 (1973).

[15] P. Leal Ferreira, Nuovo Cim. 20, 157 (1977).

[16] P. Leal Ferreira, J.A. Helayel and N. Zagury, Nuovo Cim. A 55, 215 (1980).

[17] P. Leal Ferreira in Topics On Cosmic Rays, Vol. 1, Eds. J. Bellandi and J. Pemmaraju, p.176-187, (1984).

[18] V.O. Rivelles and F. M. Witkowski, Rev. Bras. Fis. 9, 357 (1979).

[19] F. M. Witkowski in Electromagnetic Properties of Hadrons in the Relativistic Quark Model, Tese de Doutorado, (1976).

[20] T. Muta in Foundations of Quantum Chromodynamics, World Scientific, Singapore, (1987).

[21] H. G. Rothe in Lattice Gauge Theories, An Introduction, World Scientific, New York, (1992).

[22] J. F. Gunion and L. F. Lee, Phys. Rev. D 12, 3583 (1975).

[23] T. Goldman and T. Yankielowickz, Phys. Rev. D 12, 2910 (1975).

[24] A. N. Mitra, Z. Phys. C 8, 25 (1981).

[25] H. C. Pauli, Phys. Rev. D 55, 2486 (1997).

[26] S. C. Brodsky, H. C. Pauli and S. S. Pinsky, Phys. Rep. 301, 299 (1998)

[27] A. De Rújula, H. Georgi and S. Glashow, Phys. Rev. Lett. 37, 785 (1975).

[28] P. N. Bogoliubov, Ann. Inst. Henri Poincare 1, 163 (1967).

[29] G. Breit, Phys. Rev. 34, 353 (1929).

[30] W. Lucha, D. Gromes and F. Shberl, Phys. Rep. 200, 127 (1991).

[31] F. Halzen and A. D. Martin in Quarks and Leptons, An Introductory Course in Modern Particle Physics, World Scientific, New York, (1984).
[32] S. Weinberg in The Quantum Theory of Fields, Cambridge University Press, New York, (1995).

[33] S. Godfrey and N. Isgur, Phys. Rev. D 32, 189 (1985).

[34] G. S. Bankova, J. Phys. G 19, 1143 (1993).

[35] C. Semay and B. Silvestre-Brac, Nucl. Phys. A 647, 72 (1999).

[36] C. Semay and B. Silvestre-Brac, Nucl. Phys. A 618, 455 (1997).

[37] D. P. Stanley and D. Robson, Phys. Rev. D 31, 180 (1980).

[38] C. Quigg and J. L. Rosner, Phys. Rep. 56, 167 (1979).

[39] S. N. Mukherjee et al, Phys. Rep. 231, 201 (1993).

[40] G. Jaczko and L. Durand, Phys. Rev. D 58, 4017 (1998).

[41] L. P. Fulcher, Phys. Rev. D 50, 447 (1994).

[42] A. Martin, Phys. Lett. B 214, 561 (1988).

[43] L. Durand and A. Gara, J. Math. Phys. 31, 2237 (1990).

[44] L. J. Nickisch, L. Durand and B. Durand, Phys. Rev. D 30, 660 (1984).

[45] T. Barnes and G. I. Ghandour, Phys. Lett. B 118, 411 (1982).

[46] E. E. Salpeter and H. Bethe, Phys. Rev. 84, 1232 (1951).

[47] E. E. Salpeter, Phys. Rev. 87, 328 (1952).

[48] M. E. Peskin and D. V. Schröder in An Introduction to Field Theory, Addison-Wesley Publishing Company, New York, (1994).

[49] D. Gromes, Nucl. Phys. B 131, 80 (1977).

[50] K. J. Miller and M. G. Olsson, Phys. Rev. D 28, 674 (1983).

[51] F. Gestezy et al, Phys. Rev. D 30, 2189 (1984).

[52] E. Eichten, K. Gottfried, T. Kinoshita et al, Phys. Rev. Lett. 34, 369 (1975).

[53] E. Eichten, K. Gottfried, T. Kinoshita et al, Phys. Rev. D 21, 203 (1980).

[54] R. K. Ellis, W. J. Stirling and B. R. Webber in $Q C D$ and Collider Physics, Cambridge University Press, U. K., (1996). 